\title{
Numerical Simulations and Tracer Studies as a Tool to Support Water Circulation Modeling in Breeding Reservoirs
}

\author{
Piotr Zima \\ Gdańsk University of Technology, Faculty of Civil and Environmental Engineering, \\ ul. G. Narutowicza 11/12, 80-233 Gdańsk, Poland, e-mail: pzima@pg.gda.pl
}

(Received October 15, 2014; revised December 16, 2014)

\begin{abstract}
The article presents a proposal of a method for computer-aided design and analysis of breeding reservoirs in zoos and aquariums. The method applied involves the use of computer simulations of water circulation in breeding pools. A mathematical model of a pool was developed, and a tracer study was carried out. A simplified model of two-dimensional flow in the form of a biharmonic equation for the stream function (converted into components of the velocity vector) was adopted to describe the flow field. This equation, supplemented by appropriate boundary conditions, was solved numerically by the finite difference method. Next, a tracer migration equation was solved, which was a two-dimensional advection-dispersion equation describing the unsteady transport of a non-active, permanent solute. In order to obtain a proper solution, a tracer study (with rhodamine WT as a tracer) was conducted in situ. The results of these measurements were compared with numerical solutions obtained. The results of numerical simulations made it possible to reconstruct water circulation in the breading pool and to identify still water zones, where water circulation was impeded.
\end{abstract}

Key words: breeding reservoir hydraulics, water circulation, tracer study, mathematical modeling, kinematical models

\section{Introduction}

An important factor in the breeding of warm-blooded aquatic animals are well-designed reservoirs. This applies to reservoirs used in zoos, dolphinariums, and seal facilities. Animals living, feeding, and excreting waste products in a limited space, influence water parameters. Practically all the warm-blooded animals have natural bacterial flora in their digestive systems, where, in addition to symbiotic bacteria, also fecal strains occur. Pools must be provided with water exchange to limit microbial growth that may adversely affect the health of their animal inhabitants. Particularly exposed to microbial growth are zones of low flow velocity, where sedimentation of the suspension process occurs, and temperature increases as a result of sunlight. These are conditions that cause an intense growth of microorganisms and algae, which may affect microbiological water parameters. These problems are associated with the 
inappropriate construction assumptions (at the design stage) concerning conditions for the circulation of water in reservoirs. They can be countered by increasing the zone of active water exchange and by creating appropriate hydraulic conditions to eliminate still water zones ("dead zones"), where water circulation is impeded. One of the methods that make it possible to identify these types of problems and improve circulation in pools is the mathematical modeling of the flow field through numerical simulations. Additionally, a tracer study may be carried out. In the case of breeding tanks, it is very important to select a tracer (marker) that does not affect the animals living there. Such a substance is rhodamine WT, commonly used in the natural environment (Parker 1973, Smart and Laidlaw 1977, Wilson et al 1986, Kilpatrick and Wilson 1989, Zima 2012). In a tracer study, dye molecules move along with water molecules and undergo a process of diffusion. The concentration of the tracer dye in space and time can serve as the basis for the testing of theoretical models (Zima 2012).

\section{Mathematical Model Development}

To construct a comprehensive model describing, in combination with tracer studies, the process of water circulation in breeding pools, it is necessary to solve two fundamental problems: to determine the hydrodynamic behavior of a mixture and to describe the unsteady transport of dissolved matter. The former concerns the description of the flow of a water-solute mixture through the reservoir. This problem can be dealt with by one of kinematical models (Sawicki 1998, 2009, Zima 2012) described by the biharmonic equation:

$$
\Delta \Omega=\Delta \Delta \psi=\frac{\partial^{4} \psi}{\partial x^{4}}+2 \frac{\partial^{4} \psi}{\partial x^{2} \partial y^{2}}+\frac{\partial^{4} \psi}{\partial y^{4}}=0
$$

where:

$\Omega(x, y)$ - harmonic function of vorticity distribution, $\psi(x, y)-$ stream function, defined as follows (Potter 1982),

$$
u_{x}=\frac{\partial \psi}{\partial y}, \quad u_{y}=-\frac{\partial \psi}{\partial x}
$$

$u_{x}, u_{y}-$ coordinates of a two-dimensional velocity vector.

$$
\boldsymbol{u}=\left[u_{x}, u_{y}\right] .
$$

The application of model (1) is known in the literature as the solution of the two-dimensional Stokes problem (Glowinski and Pironneau 1977). The omission of the impact of inertia forces in a general description of fluid motion is obviously passed on to the scope of such a model in practical applications. If we assume that the operator $\Omega$ refers to a two-dimensional area bounded by the edge $\Gamma$ and with the three 
functions $f, g_{1}$ and $g_{2}$, in the general case, we consider the Dirichlet problem for the biharmonic operator:

$$
\Delta \Omega=\left\{\begin{array}{c}
\Delta^{2} \psi=f \\
\left.\psi\right|_{\Gamma}=g_{1} \\
\frac{\partial \psi}{\partial n}=g_{2}
\end{array}\right\} .
$$

The solution of problem (4) in two-dimensional space $(x, y)$ is a stream function $\psi(x, y)$. In this case, the function $f=0$. Next, the components of the velocity vector $\boldsymbol{u}$ (3) can be calculated in the whole domain with the use of relation (2).

The transport of the solute component $i$ in the two-dimensional approach in which the velocity field is described by vector (3), for a non-active, permanent substance (tracer), can be described by the following equation (Crank 1975, Rutherford 1994, Sawicki 2003):

$$
\frac{\partial c_{i}}{\partial t}+\frac{\partial\left(u_{x} c_{i}\right)}{\partial x}+\frac{\partial\left(u_{y} c_{i}\right)}{\partial y}=\frac{\partial}{\partial x}\left(D_{x x} \frac{\partial c_{i}}{\partial x}+D_{x y} \frac{\partial c_{i}}{\partial y}\right)+\frac{\partial}{\partial y}\left(D_{y x} \frac{\partial c_{i}}{\partial x}+D_{y y} \frac{\partial c_{i}}{\partial y}\right)
$$

where:

$$
\begin{aligned}
& c_{i} \quad-\text { concentration of the } i \text { component (substance), } \\
& D_{x x}=D_{L} n_{x}^{2}+D_{H} n_{y}^{2}, \quad D_{x y}=D_{y x}=\left(D_{L}-D_{H}\right) n_{x} n_{y}, \quad D_{y y}=D_{L} n_{y}^{2}+D_{H} n_{x}^{2}, \\
& \text { are described by Elder formulas (Elder 1959): } \\
& D_{L}=5.93 \cdot H v^{*}, \quad D_{H}=0.23 \cdot H v^{*}, \\
& \text { n } \quad-\text { directional velocity vector, } \\
& n_{x}=\frac{u_{x}}{|\boldsymbol{u}|}, \quad n_{y}=\frac{u_{y}}{|\boldsymbol{u}|}, \\
& v^{*} \quad-\quad \text { dynamic velocity (Taylor 1954). }
\end{aligned}
$$$$
D_{L}, D_{H}-\text { longitudinal and horizontal coordinates of the dispersion tensor }
$$

Relations (1) and (5) are partial differential equations, which in the general case can be solved only by numerical methods, such as the finite difference method (FDM), finite element method (FEM), or finite volume method (FVM) (Abbott 1979, Fletcher 1991, LeVeque 2002, Szymkiewicz 2000). In this study, the FDM was used to solve the biharmonic equation (Kosma 2009), and the FVM was applied to unsteady transport equations (LeVeque 2002). In order to apply these methods, the domain was divided 


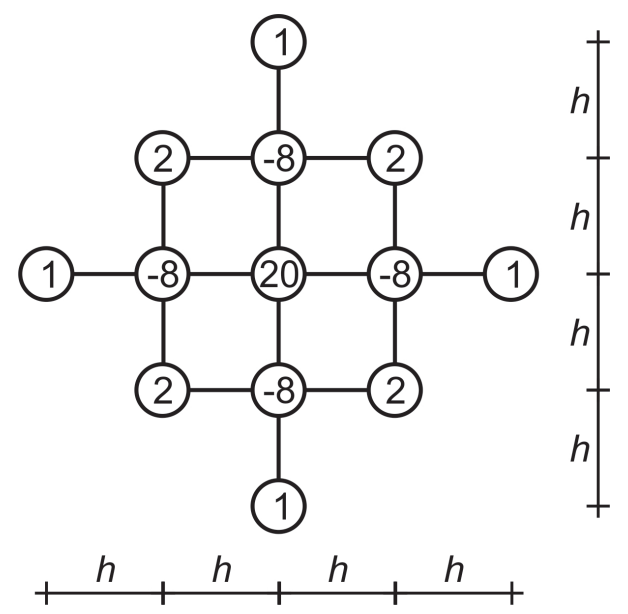

Fig. 1. A thirteen-point scheme used for the approximation of the biharmonic equation

into square elements (cells) where $\Delta x=\Delta y=h$ (Fig. 1), with $n$ nodes in direction $x$ and $m$ nodes in direction $y$.

In the case of the biharmonic equation, as a result of the approximation of the difference quotients, the equation is obtained in each computational node. Fig. 1 shows the values of the coefficients in each node $(i, j)$ resulting from the adopted approximation of equation (1) patterns of the central differential schemes (the thirteen-point scheme). In the case of boundary nodes, the above scheme was changed according to the specified boundary condition, in accordance with (4). These equations are linear and form a system that can be solved by one of the known iterative methods. In this paper, the relaxation method (the Gauss-Seidel method, also known as the Liebmann method or the method of successive displacement) was adopted (Kosma 2009):

$$
\psi_{i, j}^{(k+1)}=\psi_{i, j}^{(k)}+r_{i, j}^{(k+1, k)}
$$

where $k$ is the number of iteration, $r_{i, j}$ is a residual of the approximation of the biharmonic equation in a given node (some nods in the thirteen-point scheme follow after iteration $k$ and some after iteration $k+1$ ). The final step is the calculation of velocity vector components in the whole domain in accordance with relation (2). The partial derivatives in equations (2) were approximated by the corresponding central differential schemes (Potter 1982):

$$
u_{x}=\frac{\psi_{i+1, j}-\psi_{i-1, j}}{2 h}, \quad u_{y}=-\frac{\psi_{i, j+1}-\psi_{i, j-1}}{2 h} .
$$

The FVM, applied to solve the unsteady transport equation, refers to the physical conservation laws at the control volume level. This can be described by the homogeneous hyperbolic equation as (LeVeque 2002):

$$
\frac{\partial c}{\partial t}+\nabla \cdot \boldsymbol{F}=0
$$


Equation (5) can be written in vector form (11). If during the flow of solute we consider advection and dispersion fluxes, described by an analog to Fick's law (Sawicki 2003), the flux $\boldsymbol{F}$ can be defined as follows (LeVeque 2002):

$$
\boldsymbol{F}=\boldsymbol{u} \cdot c-D \cdot \nabla c
$$

As a result of integrating equation (11) in each finite volume and applying the Gauss-Ostrogradsky theorem, we obtain the following equation (LeVeque 2002):

$$
\frac{\partial c_{i}}{\partial t} \Delta A_{i}+\oint_{L_{i}}(\boldsymbol{F} \cdot \boldsymbol{n}) d L=0
$$

where $\Delta A_{i}$ and $L_{i}$ are, respectively, the surface and edge of the cell, and the flux $\boldsymbol{F}$ is defined by equation (12). This integral represents the advection-dispersion mass flux of substances carried by the edge of the cell. When using square elements, each of the integrals occurring in equation (13) can be replaced by the sum of four components, which makes it possible to write equation (13) in the form:

$$
\frac{\partial c_{i}}{\partial t} \Delta A_{i}+\sum_{r=1}^{4}\left(\boldsymbol{F}_{r} \cdot \boldsymbol{n}_{r}\right) \Delta L_{r}=0
$$

where $\boldsymbol{F}_{r}$ is a vector of streams by the edge $r$, and $\Delta L_{r}$ is the length of the edge of the cell. In this paper, a regular square grid was used, compatible with the directions of the Cartesian coordinate system axes (Fig. 2). This grid referred to the differential grid used to solve the biharmonic equation (Fig. 1).

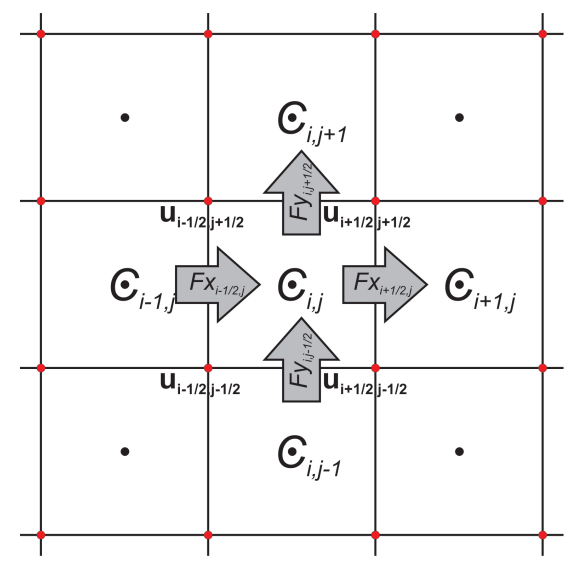

Fig. 2. The division of the domain into two-dimensional cells and the adopted parameters of the model used in the solution of the unsteady solute transport equation 
Therefore, streams by the edge in equation (14) will also be in line with the directions of the coordinate system $(x$ and $y$ ). The form of flux vector components by the edges $x$ and $y$ will be as follows:

$$
\begin{aligned}
& F_{x}=F_{A x}+F_{D x}=u_{x} \cdot c-D_{x x} \cdot \frac{\partial c}{\partial x}-D_{x y} \cdot \frac{\partial c}{\partial y}, \\
& F_{y}=F_{A y}+F_{D y}=u_{y} \cdot c-D_{y y} \cdot \frac{\partial c}{\partial y}-D_{x y} \cdot \frac{\partial c}{\partial x} .
\end{aligned}
$$

To solve equation (5), the splitting technique was used (LeVeque 2002). The transport of dissolved matter was split into autonomous processes: advection and dispersion. To determine numerical fluxes by the cell edge in the advection equation, the Lax-Wendroff scheme was used (LeVeque 2002). In the case of the dispersion equation, central differential schemes were used. In the method of integrating equations (15) and (16), the control area was assumed to be equivalent to a grid cell. As a result of this approach, the values of the velocity vector $\boldsymbol{u}$ (3) are given at grid points, and the unknown are the functions located at the central points of cells (Fig. 2). Intermediate values that lie between grid nodes (which are needed to determine the value of fluxes by individual cell edges) were calculated by averaging neighboring values. This approach was applied to the function of concentration and the components of the velocity vector.

\section{Tracer Study}

Studies on solute transport in an aquatic environment often require the adopted mathematical models to be verified and validated (Tucker 1995). Water tracing by dye fluorometry is the most common method by which data are collected for these purposes, and such studies are aimed at determining the characteristics of water movement (Zima 2012).

An extensive use of fluorescent dyes as water tracers dates back to the early $1960 \mathrm{~s}$ (Pritchard and Carpenter 1960). Over the last several decades, only two dyes (variations of the same molecular structure called xanthene), have been used as fluorescent substances in such studies: intracid rhodamine B and rhodamine WT. Rhodamine WT is preferred for most fluorometry studies because of its ease of use, relatively low cost, low adsorption tendency, strong fluorescent properties, high diffusivity, chemical stability, and limited impact on the aquatic environment (Parker 1973, Smart and Laidlaw 1977, Wilson et al 1986, Kilpatrick and Wilson 1988). In addition, the spectral characteristics of rhodamine WT are quite different from those of most other substances in the surrounding aqueous environment, making it ideal for use as a marker in tracer studies of water and wastewater (Wilson et al 1986, Zima 2012).

This article presents the results of a tracer study carried out in situ in a facility with breeding pools. The dimensions and shape of the reservoir, as well as the location of the water inlet and outlet, are presented in Fig. 3. A twenty-percent solution of 


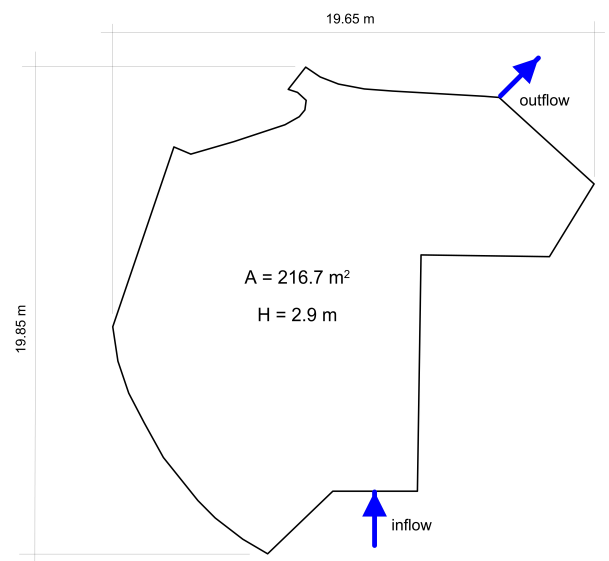

Fig. 3. Dimensions and shape of the reservoir and the location of the water inlet and outlet in the tested object

rhodamine WT was used as a tracer. The tracer was injected into the pool at a point very close to the water inlet, and concentration measurements were made near the water outlet (Fig. 4). The concentration of the tracer was measured with a submersible probe Cyclops-7 (Turner Designs) for continuous measurement of rhodamine WT concentration (Fig. 4).

a)

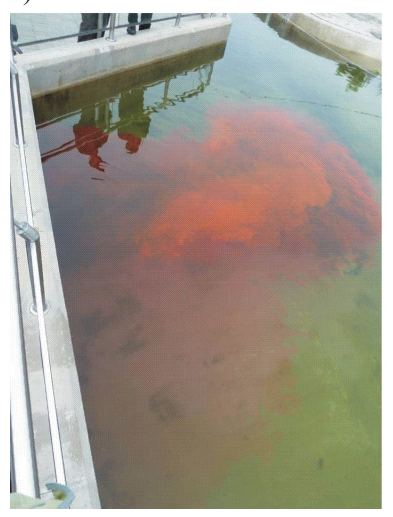

b)

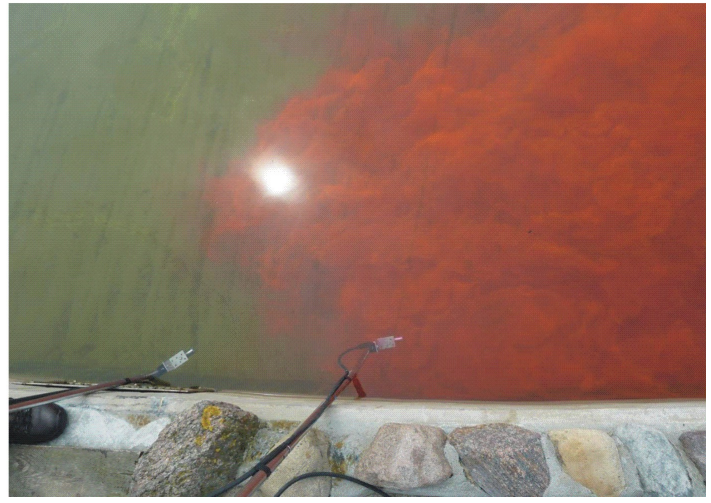

Fig. 4. The injection of tracer close to the inlet (a) and the making of measurements near the outlet (b) of the reservoir

The results of measurement (rhodamine WT concentration in percent in relation to the maximum measuring range of the probe) after 21 minutes from injection are presented in Fig. 5. It shows that the time of arrival of the first doses of the tracer at the outlet was approx. 7 minutes. It was one of the basic parameters compared with the results obtained during the numerical simulation. 


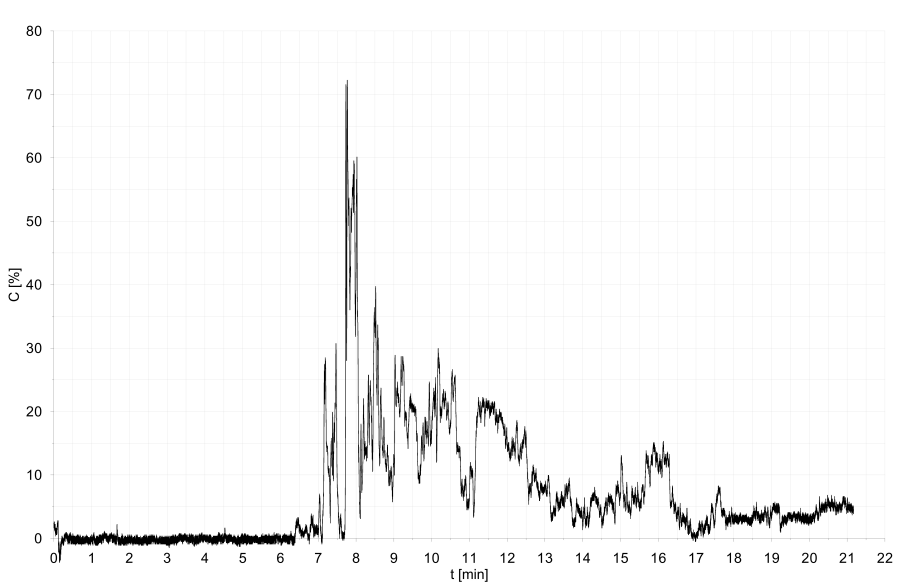

Fig. 5. The results of tracer concentration measurement after 21 minutes

\section{Numerical Calculations and Discussion of Results}

On the basis of the in-situ measurements, numerical simulations were made. Mathematical models (described in section 2 of this paper) were used to simulate flow in a model body of water. The example refers to a real-life reservoir (a breeding pool for warm-blooded animals). The reservoir has an approximately constant depth $(H=2.9$ $\mathrm{m})$. At its extreme ends, the inlet and outlet of water are installed in order to ensure the necessary exchange of water (design intent). The shape of the tank contour and the location of the inlet and outlet are shown in Fig. 3. In order to obtain the velocity vector $u$ (3) distribution for steady flow, the domain limited by the black contour was covered with a square grid of side $h=0.1 \mathrm{~m}$, and then the biharmonic equation was solved by the formula of the relaxation method (9). Equation (1) is a fourth-order elliptic differential equation, which requires two boundary conditions (Meerschaert 2007):

- Dirichlet condition - known value of the stream function, where $\psi=$ const at an impervious boundary and $\psi=$ varians at a pervious boundary;

- Neumann condition at an impervious boundary:

$$
\frac{\partial \psi}{\partial n}=0
$$

The inflow and outflow of water were appropriately included in the mathematical model as boundary conditions (linear change of the stream function at those locations). The results of computer simulations (distribution of stream lines) are shown in Fig. 6. The stream function values are scaled there: $100 \%$ means the maximum value of the stream function $\left(33.8 / 2.9 \mathrm{~m}^{3} / \mathrm{h} / \mathrm{m}\right)$. Velocity vectors were determined by formula (2) and scaled relative to the maximum speed $0.25 \mathrm{~m} / \mathrm{s}$. When analyzing the distribution of the stream function within the outline of the domain (reservoir), one 
finds zones in which lines form closed areas. They represent areas in which the exchange of water is difficult, and its velocity declines rapidly. Especially in the corners (even open ones), still water zones can be seen, where water circulation is impeded. In these zones the accumulation of pollutants and the proliferation of pathogenic microorganisms may occur. This may affect the health of animals living in the pool. This effect was also observed during the tracer study, in which a discoloration of water, associated with increased accumulation of the tracer, was visible at those places.

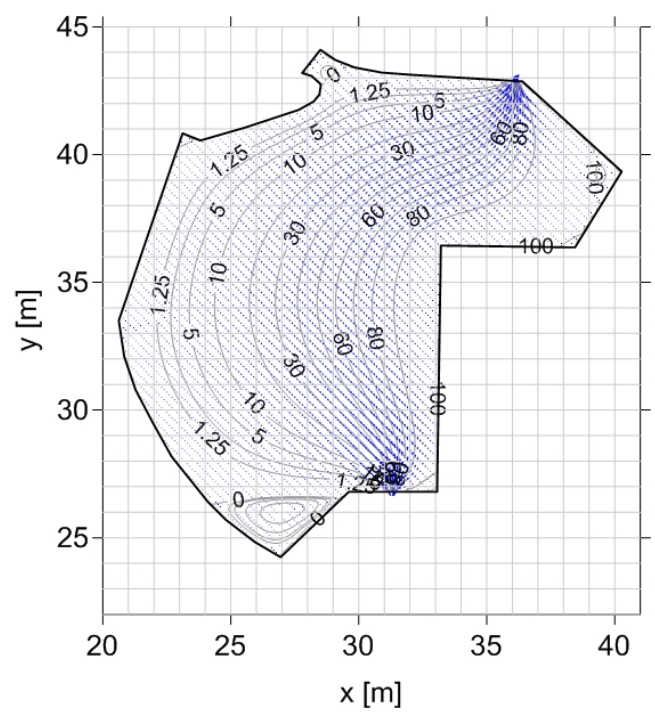

Fig. 6. Distribution of stream lines and velocity vector components in the reservoir

As the next step, in order to reconstruct the tracer flow and confirm the effects observed during the tracer study, the unsteady transport of the non-active, permanent solute component in the two-dimensional approach was simulated in a steady velocity field. The advection-dispersion equation (5) was solved by the FVM (LeVeque 2002). This is a partial differential equation of the second order, which requires the following additional conditions (Meerschaert 2007):

- initial condition - the tracer concentration $c(x, y)$ at the initial of time computing (in this case $c=0.0$ was assumed);

- boundary condition at the impermeable edge of the domain (Neumann condition) in the form:

$$
\frac{\partial c}{\partial n}=0
$$

- boundary condition at the permeable edge of the domain (Dirichlet condition) in the form:

$$
c=\text { const }
$$


a) after 2 minutes

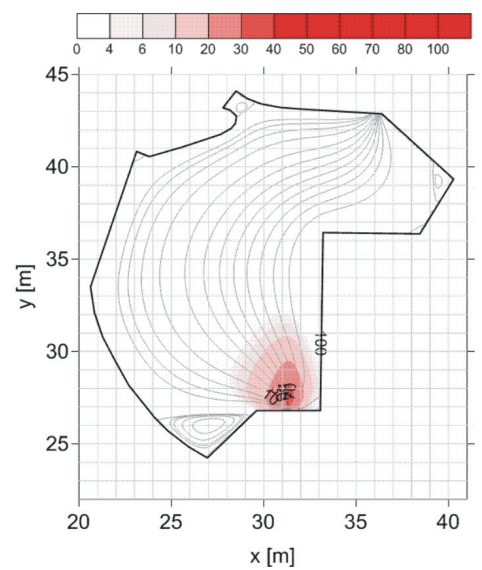

c) after 4 minutes

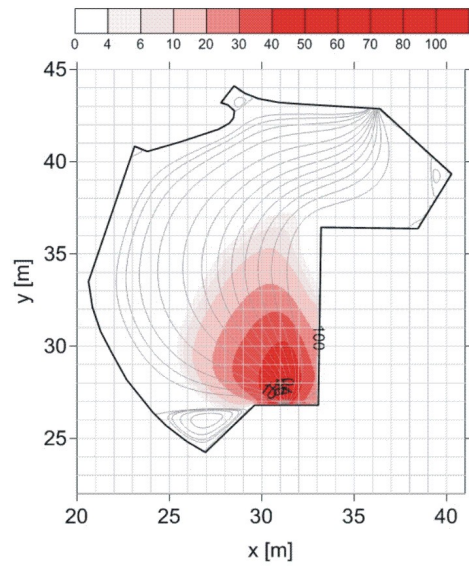

e) after 6 minutes

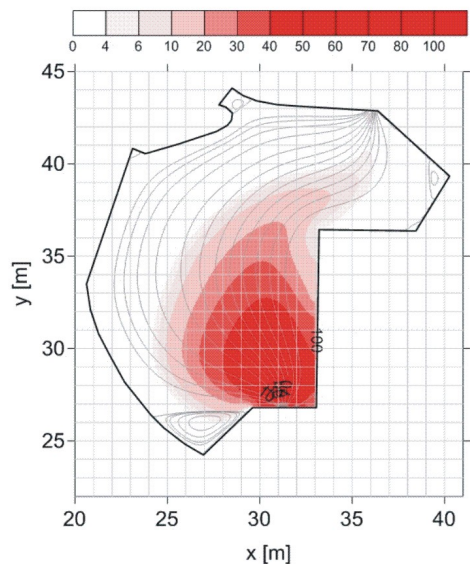

b) after 3 minutes

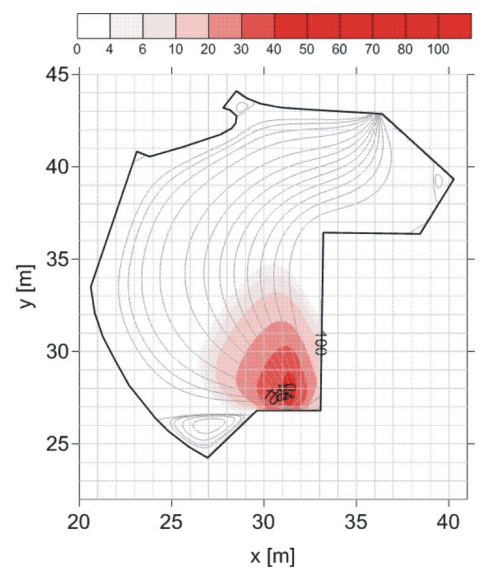

d) after 5 minutes

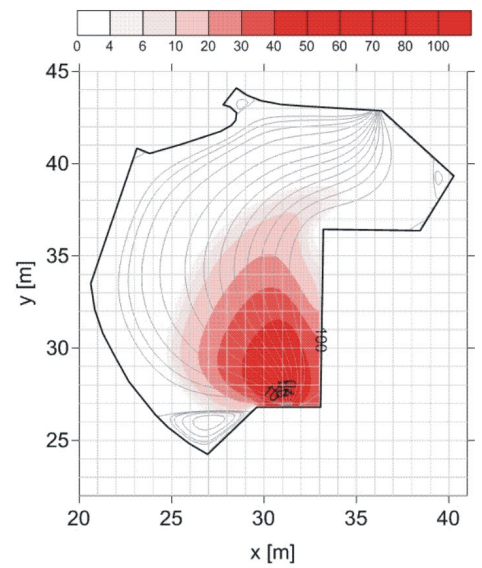

f) after 7 minutes

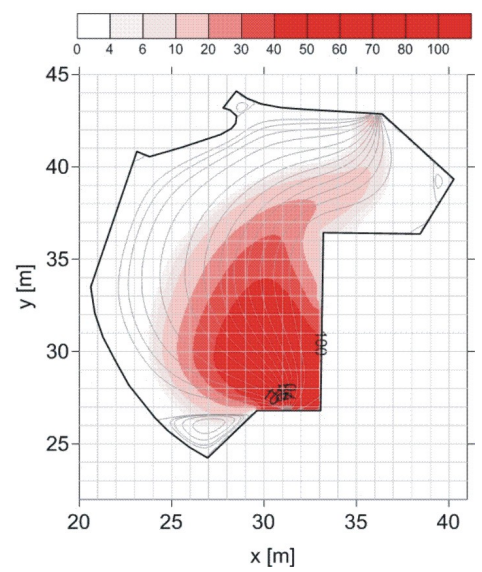

Fig. 7. Numerical simulation of tracer propagation in time and reservoir space 
In the mathematical model, at the point of the tracer input, the tracer concentration was constant and equal to the concentration of the dose injected at the inlet. This condition was enforced in the mathematical model by the Dirichlet-type condition (19) at the point of tracer injection. The results (at selected time steps) are shown in Fig. 7, which presents the distribution of the tracer in the reservoir after a specified time, from the start of the simulation until first tracer doses reached the outlet. Thus it is possible to analyze the spreading of the tracer in the entire volume of the tank and its accumulation in still water zones.

The measurment of the time of arrival of the tracer at the outlet made it possible to analyse concentration changes in time and at the measurement location. Fig. 8 presents the measurement results recorded during the tracer study (solid line), carried out as described in section 3 of this paper (Fig. 5), and the results of numerical simulation (dashed line) exactly at the point of measurement. These results may confirm the correlation between observations and the results of computer simulations, and provide a validation of the adopted mathematical model.

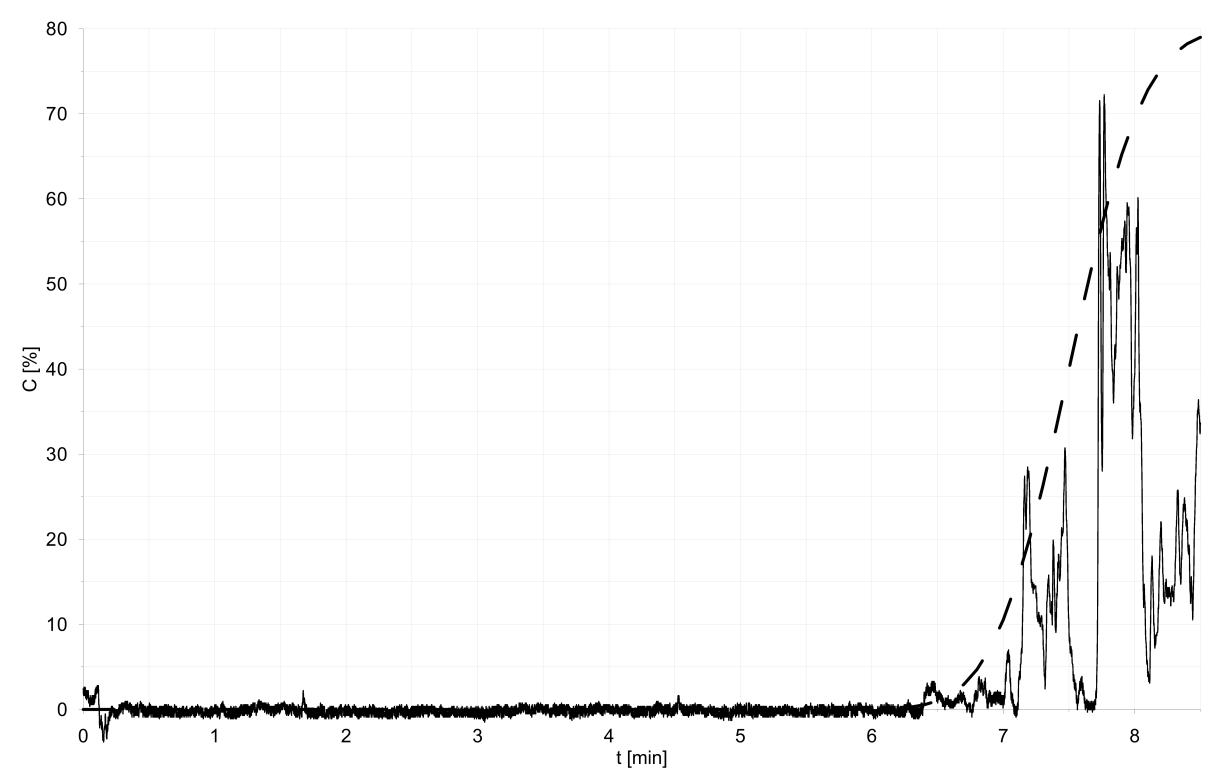

Fig. 8. Numerical simulation of tracer propagation in time at the reservoir outflow (solid line - measurements, dashed line - calculations)

\section{Conclusions}

The studies conducted for this paper were aimed at the identification of the hydraulic conditions of water circulation in breeding reservoirs, such as pools. The tests were performed by means of a tracer study in situ and by mathematical modeling. Computer simulations of the stream function and velocity field distribution under steady 
flow conditions were performed. A kinematic model in the form of the biharmonic equation was used for calculations. In addition, a tracer study was conducted, in which rhodamine WT was used as the tracer. In order to reconstruct the tracer flow and confirm the effects observed during the tracer study, the unsteady transport of a non-active, permanent solute component in the two-dimensional approach was simulated in a steady velocity field. The results obtained suggest that the models applied in the study well describe the main features of the flow related to the distribution of velocity, reconstruction of water circulation in pools, and identification of still water zones, where water circulation is impeded. Therefore, it may be advisable to apply the proposed approach in developing design guidelines for this type of breeding facilities and to use the results of this study in the modernization of such facilities for aquatic animals, e.g. in zoos, seal facilities, or fish ponds.

\section{References}

Abbott M. B. (1979) Computational hydraulics: elements of the theory of free-surface flows, Pitman, London.

Crank J. (1975) The mathematics of diffusion, Oxford: Clarendon Press.

Elder J. W. (1959) The dispersion of marked fluid in turbulent shear flow, J. of Fluid Mech., 5 (4), 544-560.

Fletcher C. A. J. (1991) Computational Techniques for Fluid Dynamics 1. Fundamental and General Techniques, Springer-Verlag, Berlin.

Glowinski R., Pironneau O. (1977) Numerical Methods For the First Biharmonic Equation and For the Two-Dimensional Stokes Problem, Raport STAN-CS-77-615, may 1977, Computer Science Department, School of Humanities and Sciences, Stanford University.

Kilpatrick F. A., Wilson J. F. (1989) Measurement of time of travel in streams by dye tracing, U.S. Geological Survey Techniques of Water Resources Investigations, Book 3, Chapter A9, 1989.

Kosma Z. (2009) Numerical methods and algorithms, Technical University of Radom Press, Radom (in Polish).

LeVeque R. J. (2002) Finite Volume Method for Hyperbolic Problems, Cambridge University Press, New York.

Meerschaert M. M. (2013) Mathematical Modeling, 4 ed., Elservier Science, Academic Press, Department of Statistics and Probability, Michigan State University, USA.

Parker G. G. (1973) Tests of rhodamine WT dye for toxicity to oysters and fish, U.S. Geological Survey Journal Research, 1 (4), p. 499.

Potter D. (1982) Computational Physics, PWN, Warsaw (in Polish).

Pritchard D. W., Carpenter J. H. (1960) Measurements of turbulent diffusion in estuarine and inshore waters, International Association of Scientific Hydrology, Bulletin, 20, 37-50.

Rutherford J. C. (1994) River Mixing, Chichester, England: Wiley.

Sawicki J. M. (1998) Free surface flows, PWN, Warsaw (in Polish).

Sawicki J. M. (2003) Migration of pollutants, Gdańsk University of Technology Press, Gdańsk (in Polish).

Sawicki J. M. (2009) Mechanics of flows, Gdańsk University of Technology Publishing, Gdańsk (in Polish).

Smart P. L., Laidlaw I. M. S. (1977) An evaluation of some fluorescent dyes for water tracing, Water Resources Research, 13 (1), 15-33. 
Szymkiewicz R. (2000) Mathematical modelling of open channel flows, PWN, Warsaw (in Polish).

Tucker W. et al (1995) A Glossary of Modeling and Simulation Terms for Distributed Interactive Simulation, www.tmpo.nima.mil/guides/Glossary/.

Taylor G. J. (1954) The dispersion of matter in turbulent flow trough a pipe, Proc. Roy. Society, 223-224, 446-468.

Wilson J. F., Cobb E. D., Kilpatrick F. A. (1986) Fluorometric procedures for dye tracing, U.S. Geological Survey Techniques of Water Resources Investigations, Book 3, Chapter A12, p.34.

Zima P. (2012) Mathematical modeling of pollution transport phenomena and technological processes in selected WWTP's objects, Gdańsk University of Technology Publishing, Gdańsk (in Polish). 